\title{
Elastic crack-tip stress field in a semi-strip
}

\author{
V. Reut, N. Vaysfeld, Z. Zhuravlova \\ Odessa Mechnikov University, Institute of Mathematics, Economics and Mechanics, Ukraine \\ reu@onu.edu.ua,vaysfeld@onu.edu.ua,r.zhuravlova@onu.edu.ua
}

\begin{abstract}
In this article the plain elasticity problem for a semi-strip with a transverse crack is investigated in different cases of boundary conditions at the semi-strip's end. Unlike many works dedicated to this subject, the fixed singularities in the singular integral equation's kernel are considered. The integral transformations' method is applied by a generalized scheme to reduce the initial problem to a one-dimensional problem. The one-dimensional problem is formulated as a vector boundary value problem which is solved with the help of matrix differential calculations and Green's matrix apparatus. The problem is reduced to solve the system of three singular integral equations. Depending on the conditions given on the short edge of the semistrip, the obtained singular integral equation can have one or two fixed singularities. A special method is applied to solve this equation in regard to the singularities existence. Hence, the system of the singular integral equations (SSIE) is solved with the help of the generalized method. The stress intensity factors (SIF) are investigated for different lengths of crack. The novelty of this work is the application of a new approach allowing the consideration of fixed singularities in the problem of a transverse crack in the elastic semi-strip. The comparison of the accuracy of numerical results during the use of different approaches to solve the SSIE is calculated.
\end{abstract}

KEYWORDS. Semi-strip; Transverse crack; Green's function; Integral Transformation; Fixed singularity.

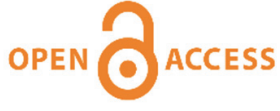

Citation: Reut, V., Vaysfeld, N., Zhuravlova, Z., Elastic crack-tip stress field in a semi-strip Frattura ed Integrità Strutturale, 44 (2018) 8293.

Received: 26.01 .2018

Accepted: 11.02 .2018

Published: 01.04.2018

Copyright: (C) 2018 This is an open access article under the terms of the CC-BY 4.0, which permits unrestricted use, distribution, and reproduction in any medium, provided the original author and source are credited.

\section{INTRODUCTION}

$\mathrm{T}$ he proposed problem is a well-known elasticity problem. It is used as a modeling example in the theory of mixed elasticity problems to recognise new methods to solve these problems. The solutions of such problems are usually reduced to SSIE, containing fixed singularities in a kernel [1]. Pioneering papers by scientists such as H. F. Bueekner, G. I. Bierman, F. Tricomi, S. G. Mihlin, M. G. Kreyn, B. Nobl and others, they first proposed different approaches for consideration of fixed singularities'.

Solving equations with fixed singularities in a kernel was made by using many methods. Analytical proof of these methods was given, for example, in [2-5]. There, the singular integral equations of the first type (one fixed singularity) 


$$
c_{0} \varphi(x)+\frac{c_{1}}{\pi i} \int_{0}^{1} \frac{\varphi(y)}{y-x} d y+\sum_{k=0}^{n} \frac{c_{k+2} x^{n_{k}}}{\pi i} \int_{0}^{1} \frac{y^{k-n_{k}} \varphi(y)}{(y+x)^{k+1}} d y=f(x), x \in I=[0 ; 1], 0 \leq \operatorname{Re} n_{k} \leq k, k=\overline{0, n}
$$

or of the second type (two fixed singularities)

$$
\begin{aligned}
A \varphi(x) & =c_{0} \varphi(x)+\frac{c_{1}}{\pi i} \int_{-1}^{1} \frac{\varphi(y)}{y-x} d y+\sum_{k=0}^{n} \frac{1}{\pi i} \int_{-1}^{1} \frac{c_{k+2}(x, y)(1-x)^{m_{k}^{+}}(1+x)^{m \bar{k}} \varphi(y)}{(1-y)^{m_{k}^{+}-k}(1+y)^{m \bar{k}^{-k}}(1-x y)^{k+1}} d y+\int_{-1}^{1} K(x, y) \varphi(y) d y= \\
& =f(x), 0 \leq \operatorname{Re} m_{k}^{+} \leq k
\end{aligned}
$$

were considered.

The approaches to solve them were proposed in the widely known work [3]. The first equation was considered by many authors, e.g. [5-11], whereas the second equation was investigated e.g. in $[12,13]$. These methodologies were used by employing two main approaches to solve elasticity problems for semi-strips with a crack: both analytical and numerical.

The analytical approach to solve the SSIE's with two fixed singularities is often connected with unknown function's expansion in the series of polynomials with corresponding weights. An SIE with two fixed singularities at the endpoints in the class of the functions bounded at the ends was analyzed in [4]. For the Chebyshev polynomials of the first kind on the right hand-side, solution of the integral equation is expressed in terms of two non-orthogonal polynomials with associated weights. Based on this new generalized spectral relation for the singular operator with two fixed singularities, an approximate solution to the complete singular integral equation is derived by recasting it as an infinite system of linear algebraic equations of the second kind.

Some problems were solved with the apparatus of the Riemann-Hilbert problem. It is worth pointing out the following papers. In [14] the problem of semi-infinite crack between two bonded dissimilar strips with the same density was considered. The boundary problem was reduced to the Riemann-Hilbert problem. The study of the algebra generated by the Cauchy singular integral operator and integral operators with fixed singularities on the unit interval was given in [15]. In [16] a polynomial collocation method was considered for the numerical solution of the Cauchy singular integral equations with fixed singularities over the interval, where the fixed singularities are supposed to be of Mellin convolution type.

The following papers were dedicated to the numerical solving of problems with similar equations, [17] In [18] the solution of a dynamic problem of an elastic strip, coupled to an elastic half-space, is reduced to a singular integral equation that is solved with the help of special quadrature formulae for singular integrals. An approach to investigate the optimal quadrature formulae for singular integrals with fixed singularity was obtained in [19]. In [20], the quadrature formulae of the highest algebraic accuracy were obtained for SSIE. The efficiency of their application in solving the singular integral equations with the generalized Cauchy kernel was showed. In [21] the new versions of subdomain and spline methods were proposed. Collocation methods were proposed in [22].

The problem of stress concentration near the crack's tips is an actual problem. Interface cracks in bodies under harmonic load was investigated in [23]. Microstructure influence on the damage micromechanisms in overloaded fatigue cracks was studied in [24]. Crack-tip field in circumferentially-cracked round bar (CCRB) in tension affected by loss of axial symmetry was explored in [25]. In [26] the overview of recent advanced methods for rapid calculation of notch stress intensity factors under mixed mode loadings was presented. The analytical approach for plane elastic and thermoelastic problems for inhomogeneous, orthotropic planes, half-planes and strips was presented in [27].

To solve mixed problems, many authors mostly introduce some auxiliary functions, for example, harmonic and byharmonic ones, through which unknown displacements are represented. Reconstruction of the initial characteristics in this case is often a non-trivial mathematic problem. In this work, the new methods based on direct application of integral transformations to the equilibrium equations is solved, so no additional transformations are needed. Thus it was possible to find directly the real mechanical characteristics without using any auxiliary functions. This approach was shown first in [28] to solve the problem of the semi-strip without the existence of a crack.

\section{STATEMENT OF THE PROBLEM}

7 he elastic ( $G$ is a share module, $\mu$ is a Poison's coefficient) semi-strip, $0<x<a, 0<y<\infty$ is considered for two cases with regard to the boundary conditions on the short edge $0<x<a, y=0$. At the lateral semi-infinite sides $x=0, \quad 0<y<\infty$ and $x=a, \quad 0<y<\infty$ the boundary conditions are given 


$$
u(0, y)=0, v(0, y)=0, u(a, y)=0, v(a, y)=0, \quad 0<y<\infty
$$

here $u(x, y)=u_{x}(x, y), v(x, y)=u_{y}(x, y)$ are the displacements that satisfy the Lame's equilibrium equations

$$
\left\{\begin{array}{l}
\frac{\partial^{2} u(x, y)}{\partial x^{2}}+\frac{\kappa-1}{\kappa+1} \frac{\partial^{2} u(x, y)}{\partial y^{2}}+\frac{2}{\kappa+1} \frac{\partial^{2} v(x, y)}{\partial x \partial y}=0 \\
\frac{\partial^{2} v(x, y)}{\partial x^{2}}+\frac{\kappa+1}{\kappa-1} \frac{\partial^{2} v(x, y)}{\partial y^{2}}-\frac{2}{\kappa-1} \frac{\partial^{2} u(x, y)}{\partial x \partial y}=0
\end{array}\right.
$$

where $\kappa=3-4 \mu$ is the Muskchelishvili's constant. Two cases of the boundary conditions on the short edge are considered. In the first case (Fig. 1) the semi-strip is loaded at the edge $y=0, \quad 0<x<a_{1}$

$$
\sigma_{y}(x, 0)=p(x), \quad \tau_{x y}(x, 0)=0, \quad 0<x<a_{1}
$$

and conditions of the slide contact are executed at the segment $y=0, a_{1}<x<a$

$$
v(x, 0)=0, \quad \tau_{x y}(x, 0)=0, \quad a_{1}<x<a
$$

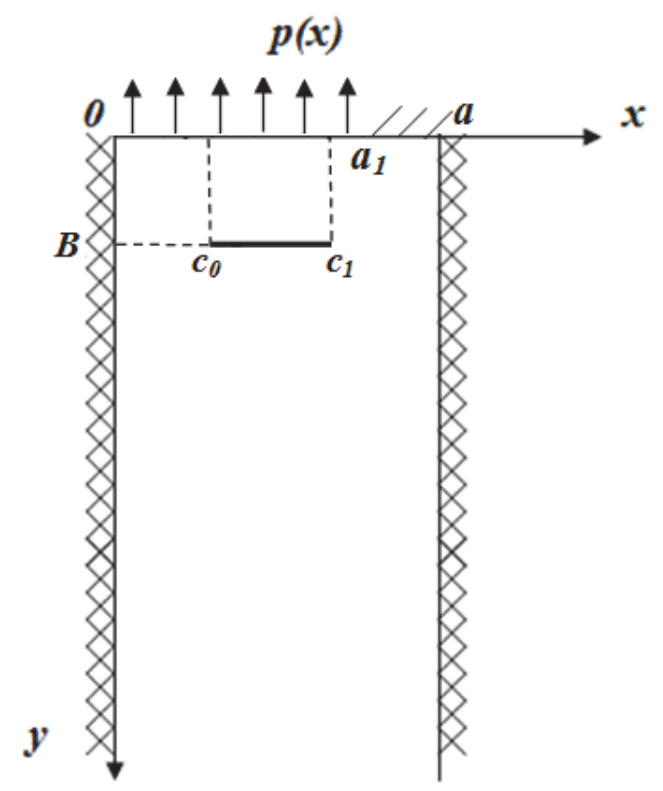

Figure 1: First case: geometry and coordinate system of the problem.

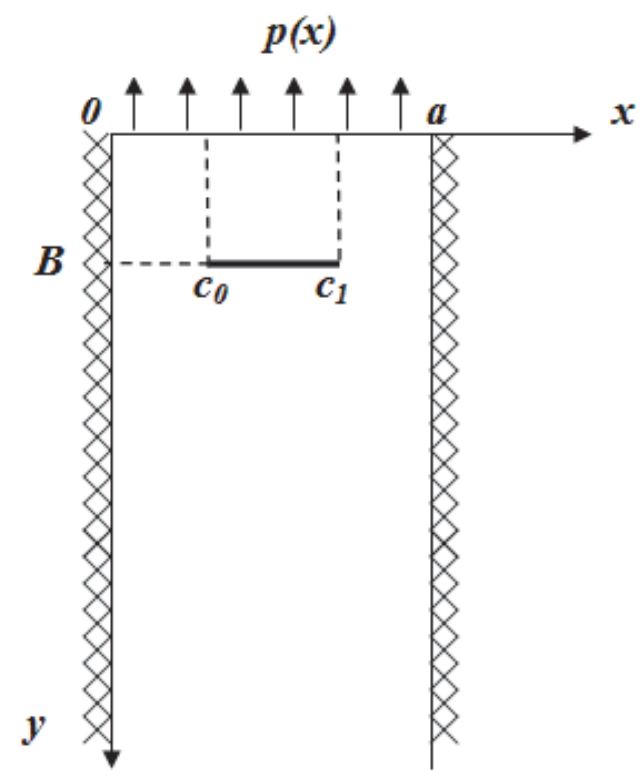

Figure 2: Second case: geometry and coordinate system of the problem.

In the second case (Fig. 2) the semi-strip is loaded at the edge $y=0, \quad 0<x<a$

$$
\sigma_{y}(x, 0)=p(x), \quad \tau_{x y}(x, 0)=0, \quad 0<x<a
$$

At the segment $c_{0}<x<c_{1}, y=B$ the crack is situated

$$
\begin{array}{ll}
u(x, B-0)-u(x, B+0)=\langle u(x, B)\rangle=\psi_{1}(x) \neq 0, & c_{0}<x<c_{1} \\
v(x, B-0)-v(x, B+0)=\langle v(x, B)\rangle=\psi_{2}(x) \neq 0, & c_{0}<x<c_{1}
\end{array}
$$




$$
\begin{array}{ll}
\tau_{x y}(x, B-0)-\tau_{x y}(x, B+0)=\left\langle\tau_{x y}(x, B)\right\rangle=0, & c_{0}<x<c_{1} \\
\sigma_{y}(x, B-0)-\sigma_{y}(x, B+0)=\left\langle\sigma_{y}(x, B)\right\rangle=0, & c_{0}<x<c_{1}
\end{array}
$$

One needs to solve the corresponding boundary value problems to estimate the stress state of the semi-strip and the concentration of the stresses at the crack's tips.

\section{GENERAL SOLVING SCHEME FOR THE SEMI-STRIP STRESS STATE ESTIMATION}

A

ccording to the approach [29], the Fourier's transformation was applied to the system of Lame's equilibrium Eqs. (2) and to the boundary conditions (1), (3)-(4), (1), (5) by the generalized scheme [30]. The initial problem was reduced to a vector boundary problem [31]

$$
\begin{aligned}
& L_{2} \vec{y}_{\beta}(x)=\vec{f}(x) \\
& \vec{y}_{\beta}(0)=0, \vec{y}_{\beta}(a)=0
\end{aligned}
$$

here $L_{2} \vec{y}_{\beta}(x)=I_{\beta}{ }^{\prime \prime}(x)+2 \beta \vec{y}_{\beta}{ }^{\prime}(x)-\beta^{2} P \vec{y}_{\beta}(x)$ is the differential operator of the second order, $I$ is an identity matrix,

$$
\begin{aligned}
& \vec{y}_{\beta}(x)=\left(\begin{array}{l}
u_{\beta}(x) \\
v_{\beta}(x)
\end{array}\right) \\
& P=\left(\begin{array}{cc}
\frac{\kappa-1}{\kappa+1} & 0 \\
0 & \frac{\kappa+1}{\kappa-1}
\end{array}\right) \\
& Q=\left(\begin{array}{cc}
0 & \frac{1}{\kappa+1} \\
-\frac{1}{\kappa-1} & 0
\end{array}\right) \\
& \vec{f}(x)=\left(\begin{array}{c}
\frac{3-\kappa}{\kappa+1} \chi^{\prime}(x)-\beta \sin \beta b \frac{\kappa-1}{\kappa+1} \psi_{1}(x)+\cos \beta b \frac{\kappa-3}{\kappa+1} \psi^{\prime}{ }_{2}(x) \\
-\beta \frac{\kappa+1}{\kappa-1} \chi(x)-\sin \beta b \psi^{\prime}{ }_{1}(x)+\beta \frac{\kappa+1}{\kappa-1} \cos \beta b \psi_{2}(x)
\end{array}\right)
\end{aligned}
$$

$\chi(x)=\left.v(x, y)\right|_{y=0}$ is an unknown function. So $\left.v^{\prime}(x, y)\right|_{y=0}=\chi^{\prime}(x), \partial u /\left.\partial y\right|_{y=0}=\chi^{\prime}(x)$, and the second boundary condition in (3) is satisfied automatically. The components of the vector $\vec{y}_{\beta}(x)$ are the Fourier transformation of the displacements

$$
\left[\begin{array}{l}
u_{\beta}(x) \\
v_{\beta}(x)
\end{array}\right]=\int_{0}^{\infty}\left[\begin{array}{l}
u(x, y) \\
v(x, y)
\end{array}\right]\left[\begin{array}{c}
\cos \beta y \\
\sin \beta y
\end{array}\right] d y
$$

The solution of the vector boundary problem was obtained in the form [27, 28] 


$$
\vec{y}_{\beta}(x)=Y_{1}(x)\left(\begin{array}{l}
c_{1} \\
c_{2}
\end{array}\right)+Y_{2}(x)\left(\begin{array}{c}
c_{3} \\
c_{4}
\end{array}\right)+\int_{0}^{a} G(x, \xi) \vec{f}(\xi) d \xi
$$

where $Y_{1}(x), Y_{2}(x)$ are the system of fundamental matrix solutions, $c_{i}, i=\overline{1,4}$ are known constants, $G(x, \xi)$ is the Green's matrix function [29].

The expression (10) can be rewritten in scalar form

$$
\begin{aligned}
& u_{\beta}(x)=Y_{1}^{11}(x) c_{1}+Y_{1}^{12}(x) c_{2}+Y_{2}^{11}(x) c_{3}+Y_{2}^{12}(x) c_{4}+\frac{3-\kappa}{\kappa+1} \int_{0}^{\vartheta} G^{11}(x, \xi) \chi^{\prime}(\xi) d \xi+ \\
& +\beta \frac{\kappa+1}{\kappa-1} \int_{0}^{\vartheta} \mathfrak{I}^{12}(x, \xi) \chi^{\prime}(\xi) d \xi-\frac{\kappa-1}{\kappa+1} \beta \sin \beta B \int_{c_{0}}^{c_{1}} G^{11}(x, \xi) \psi_{1}(\xi) d \xi+\sin \beta B \int_{c_{0}}^{c_{1}} \frac{\partial G^{12}}{\partial \xi}(x, \xi) \psi_{1}(\xi) d \xi- \\
& -\cos \beta B \frac{\kappa-3}{\kappa+1} \int_{c_{0}}^{c_{1}} \frac{\partial G^{11}}{\partial \xi}(x, \xi) \psi_{2}(\xi) d \xi+\beta \frac{\kappa+1}{\kappa-1} \cos \beta B \int_{c_{0}}^{c_{1}} G^{12}(x, \xi) \psi_{2}(\xi) d \xi \\
& v_{\beta}(x)=Y_{1}^{21}(x) c_{1}+Y_{1}^{22}(x) c_{2}+Y_{2}^{21}(x) c_{3}+Y_{2}^{22}(x) c_{4}+\frac{3-\kappa}{\kappa+1} \int_{0}^{\vartheta} G^{21}(x, \xi) \chi^{\prime}(\xi) d \xi+ \\
& +\beta \frac{\kappa+1}{\kappa-1} \int_{0}^{\vartheta} \mathfrak{J}^{22}(x, \xi) \chi^{\prime}(\xi) d \xi-\frac{\kappa-1}{\kappa+1} \beta \sin \beta B \int_{c_{0}}^{c_{1}} G^{21}(x, \xi) \psi_{1}(\xi) d \xi+\sin \beta B \int_{c_{0}}^{c_{1}} \frac{\partial G^{22}}{\partial \xi}(x, \xi) \psi_{1}(\xi) d \xi- \\
& -\cos \beta B \frac{\kappa-3}{\kappa+1} \int_{c_{0}}^{c_{1}} \frac{\partial G^{21}}{\partial \xi}(x, \xi) \psi_{2}(\xi) d \xi+\beta \frac{\kappa+1}{\kappa-1} \cos \beta B \int_{c_{0}}^{c_{1}} G^{22}(x, \xi) \psi_{2}(\xi) d \xi
\end{aligned}
$$

here $\mathfrak{I}^{i j}(x, \xi)=\int G^{i j}(x, \xi) d \xi$, and upper limit of the integrals $\vartheta=a_{1}$ in the first case and $\vartheta=a$ in the second case.

The inverse transformations were applied to the formulae (11)-(12), and the substitution of the displacement functions in the boundary conditions $\sigma_{y}(x, 0)=p(x), \tau_{x y}(x, B+0)=0, \sigma_{y}(x, B+0)=0$ reduce to the system of the singular integral equations.

\section{SOLVING OF THE SIE SYSTEM FOR THE TWO CASES}

7 he changing of the variable $\xi=\frac{2 \xi^{*}-\vartheta}{\vartheta}$ in the integrals with the limits 0 and $\vartheta$, and $\xi=\frac{2 \xi^{*}-\left(c_{0}+c_{1}\right)}{c_{1}-c_{0}}$ in the integrals with the limits $c_{0}$ and $c_{1}$ were done to pass the integration interval $I_{1}=[-1 ; 1]$. Similar changes were done in the other equations.

We first consider in details the second case. SSIE is written in the form

$$
\left\{\begin{aligned}
\int_{-1}^{1} \tilde{\chi}(\xi) & {\left[\frac{1}{\xi-x}+Z(x, \xi)\right] d \xi+K_{0}(x)=\tilde{r}(x), x \in I_{1} } \\
& \int_{-1}^{1} \tilde{\psi}_{1}(\xi) \frac{1}{\xi-x} d \xi+K_{1}(x)=0, x \in I_{1} \\
& \int_{-1}^{1} \tilde{\psi}_{2}(\xi) \frac{1}{\xi-x} d \xi+K_{2}(x)=0, x \in I_{1}
\end{aligned}\right.
$$


where

$$
\begin{aligned}
& \tilde{\chi}(\xi)=\chi^{\prime}\left(\frac{\vartheta(\xi+1)}{2}\right) \\
& \tilde{\psi}_{i}(\xi)=\psi_{i}^{\prime}\left(\frac{\left(c_{1}-c_{0}\right) \xi+\left(c_{1}+c_{0}\right)}{2}\right), i=1,2 \\
& K_{i}(x)=\int_{-1}^{1} \tilde{\chi}(\xi) \tilde{f}_{i}(\xi, x) d \xi+\int_{-1}^{1} \tilde{\psi}_{1}(\xi) \tilde{R}_{i, 1}(x, \xi) d \xi+\int_{-1}^{1} \tilde{\psi}_{2}(\xi) \tilde{R}_{i, 2}(x, \xi) d \xi, i=0,1,2
\end{aligned}
$$

$\tilde{f}_{i}(\xi, x), \tilde{R}_{i, 1}(x, \xi), \tilde{R}_{i, 2}(x, \xi), \tilde{r}(x), i=0,1,2$ are known regular functions,

$$
\begin{aligned}
& \mathrm{Z}(x, \xi)=h_{1}\left(\frac{1}{\xi+x-2}+\frac{1}{\xi+x+2}\right)+h_{2}\left(\frac{x-1}{(\xi+x-2)^{2}}+\frac{x+1}{(\xi+x+2)^{2}}\right)+h_{3}\left(\frac{(\xi-1)(x-1)}{(\xi+x-2)^{3}}+\frac{(\xi+1)(x+1)}{(\xi+x+2)^{3}}\right) \\
& h_{1}=-\frac{\kappa^{2}-3}{2 \kappa}, h_{2}=-\frac{2}{\kappa}, h_{3}=\frac{4}{\kappa}
\end{aligned}
$$

In this article the misprint in [28] for the coefficients $b_{i}, i=1,2,3$ is corrected.

The first singular integral equation in the system (13) is the partial case of the equation with two fixed singularities for the second case. For this equation the transcendental equation was built, that is congruent to the transcendental equation obtained for the quarter plane or, the same, for the problem of an infinity wedge when the angle of openness is pi/2 [32]. The problem for the quarter plane is solved in Appendix A. The roots $\lambda_{k}$ of the corresponding transcendental equation for (13) were found numerically.

The generalized method developed in [28] was applied to solve the SSIE (13). According to it the function $\tilde{\chi}(\xi)$ is searched in the form

$$
\tilde{\chi}(\xi)=\sum_{k=0}^{N-1}\left[s_{k}^{0} \rho_{k}^{-}(\xi)+s_{k+N}^{0} \rho_{k}^{+}(\xi)\right], \quad \xi \in[-1 ; 1]
$$

where

$$
\begin{aligned}
& \rho_{2 k}^{\mp}(\xi)=(1 \pm \xi)^{\operatorname{Re} \lambda_{k}} \cdot \cos \left(\operatorname{Im} \lambda_{k} \ln (1 \pm \xi)\right), \\
& \rho_{2 k+1}^{\mp}(\xi)=(1 \pm \xi)^{\operatorname{Re} \lambda_{k}} \cdot \sin \left(\operatorname{Im} \lambda_{k} \ln (1 \pm \xi)\right),
\end{aligned}
$$

are the unknown coefficients.

It is supposed that the crack is located inside the semi-strip far from the lateral sides. So the unknown functions $\tilde{\psi}_{1}(\xi), \tilde{\psi}_{2}(\xi)$ are considered as

$$
\tilde{\psi}_{i}(\xi)=\sum_{k=0}^{2 N-1} s_{k}^{i} \sqrt{1-\xi^{2}} U_{k}(\xi), \quad \xi \in[-1 ; 1], i=1,2
$$

where $U_{k}(\xi)$ are Chebyshev polynomials of the second kind.

The expressions (14)-(15) are substituted in the SSIE (13). The resulting system is solved with the help of the collocation method. The substitution of the founded constants $s_{k}^{i}, i=0,1,2, k=\overline{0,2 N-1}$ in the formulae (14)-(15) and (11)-(12) 
enables to find the searched field of stresses and displacements. It completes the construction of the problem's solution in the second case.

In the first case, the first equation in (13) has only one fixed singularity. Similarly to the previous case the unknown function $\tilde{\chi}(\xi)$ is searched in the form [28].

$$
\tilde{\chi}(\xi)=\sum_{k=0}^{N-1}\left[s_{k}^{0} \rho_{k}^{-}(\xi)+s_{k+N}^{0} \frac{T_{k}(\xi)}{\sqrt{1-\xi}}\right], \quad \xi \in[-1 ; 1]
$$

where $T_{k}(\xi)$ are Chebyshev polynomials of the first kind.

The formulae (15)-(16) are substituted in the SSIE (13) and the collocation method was applied to the solve the resulting system. In the first case, the construction of the problem's solution was completed by the substitution of the obtained constants $s_{k}^{i}, i=0,1,2, k=\overline{0,2 N-1}$ into the expressions (14)-(15) and (11)-(12).

\section{NUMERICAL RESULTS AND DISCUSSION}

7 he calculation for SIF was done by the formulae [30], [33]

$$
\begin{aligned}
K_{I-} & =\sum_{k=0}^{2 N-1} s_{k}^{2} \frac{\sqrt{\pi\left(c_{1}-c_{0}\right)}(n+1)(-1)^{k}}{\sqrt{2}}, K_{I+}=\sum_{k=0}^{2 N-1} s_{k}^{2} \frac{\sqrt{\pi\left(c_{1}-c_{0}\right)}(n+1)}{\sqrt{2}}, \\
K_{I I-} & =\sum_{k=0}^{2 N-1} s_{k}^{1} \frac{\sqrt{\pi\left(c_{1}-c_{0}\right)}(n+1)(-1)^{k}}{\sqrt{2}}, K_{I I+}=\sum_{k=0}^{2 N-1} s_{k}^{1} \frac{\sqrt{\pi\left(c_{1}-c_{0}\right)}(n+1)}{\sqrt{2}}
\end{aligned}
$$

The calculations of SIF were done for the elastic semi-strip $\left(G=61.2781955 \cdot 10^{9} \mathrm{~Pa}, \mu=0.33\right)$ with the parameters $p(x)=1 \mathrm{~Pa}, a=10 \mathrm{~m}, B=a, a_{1}=90 \% a$. Here $K_{I-}, K_{I+}$ are SIF of normal stresses at the left and right crack's tips correspondingly. Similarly, $K_{I I-}, K_{I I+}$ are SIF of the tangential stresses at the left and right crack's tips.
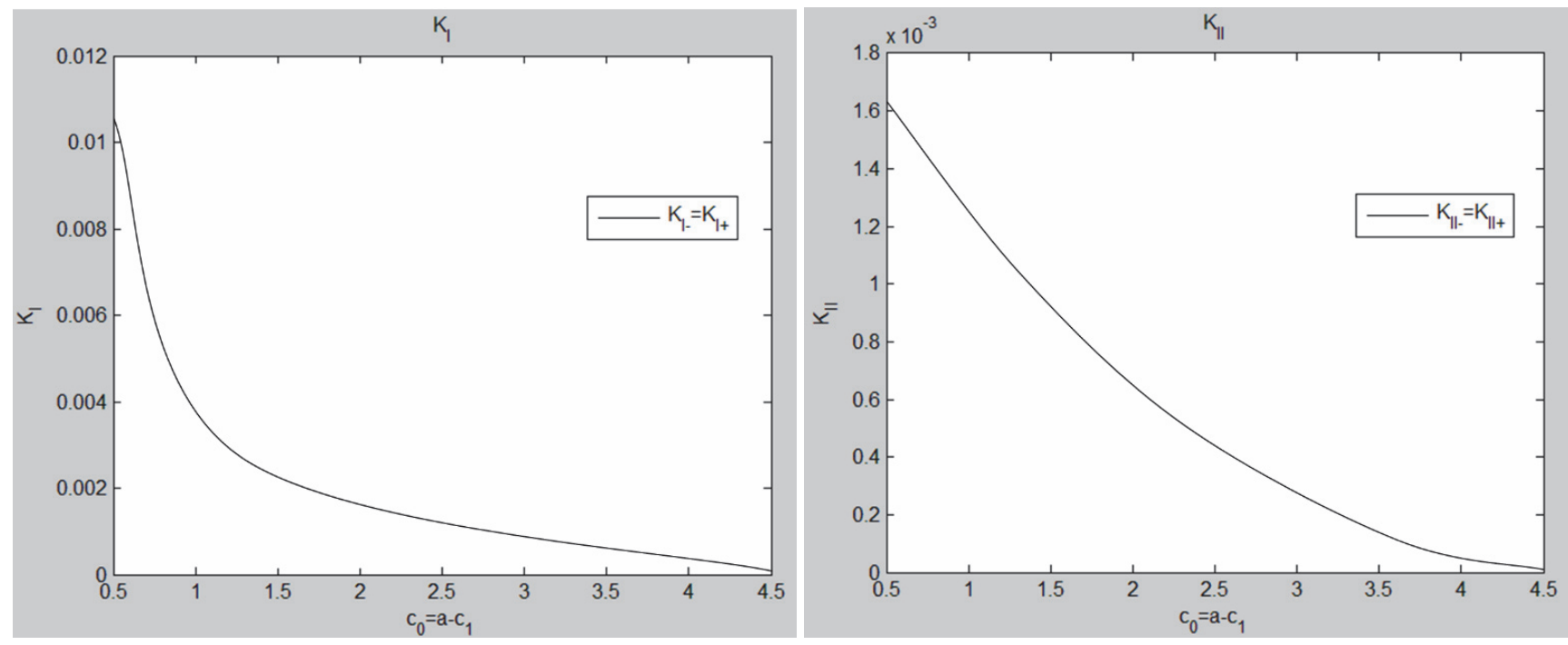

Figure 3: Second case: the changing of SIF $K_{I}, K_{I I}$ when the crack's size is increasing.

Fig. 3 presents the dynamics of SIF's changes $K_{I}$ and $K_{I I}$ respectively in dependence of the distances between the crack tips and the lateral sides of the semi-strip in the second case. The SIF values are decreasing whereas the distances between the lateral sides and the crack tips are increasing. Stable results were obtained when the distances between the crack's tips 
and the lateral sides are more or equal to $5 \% a$. When these distances are less than $5 \% a$ the fixed singularities at the crack's tips should be taken into consideration. The values of SIF $K_{I}$ are bigger than the corresponding values of SIF $K_{I I}$.

The dynamics of the SIF's changes $K_{I}$ and $K_{I I}$ respectively is shown in Fig. 4 as a function of the distances between the crack tips and the lateral sides of the semi-strip in the first case. When the distance between the left lateral side and the left crack's tip is less than $5 \% a$, the fixed singularity at the left crack's tip should be considered. The fixed singularity at the right crack's tip should be taken into account when the distance between the right lateral side and the right crack's tip is less than $11 \% a$. In this case, the SIF $K_{I}$ and $K_{I I}$ values have the same order, but the values of SIF $K_{I I}$ are bigger than the values of SIF $K_{I}$.
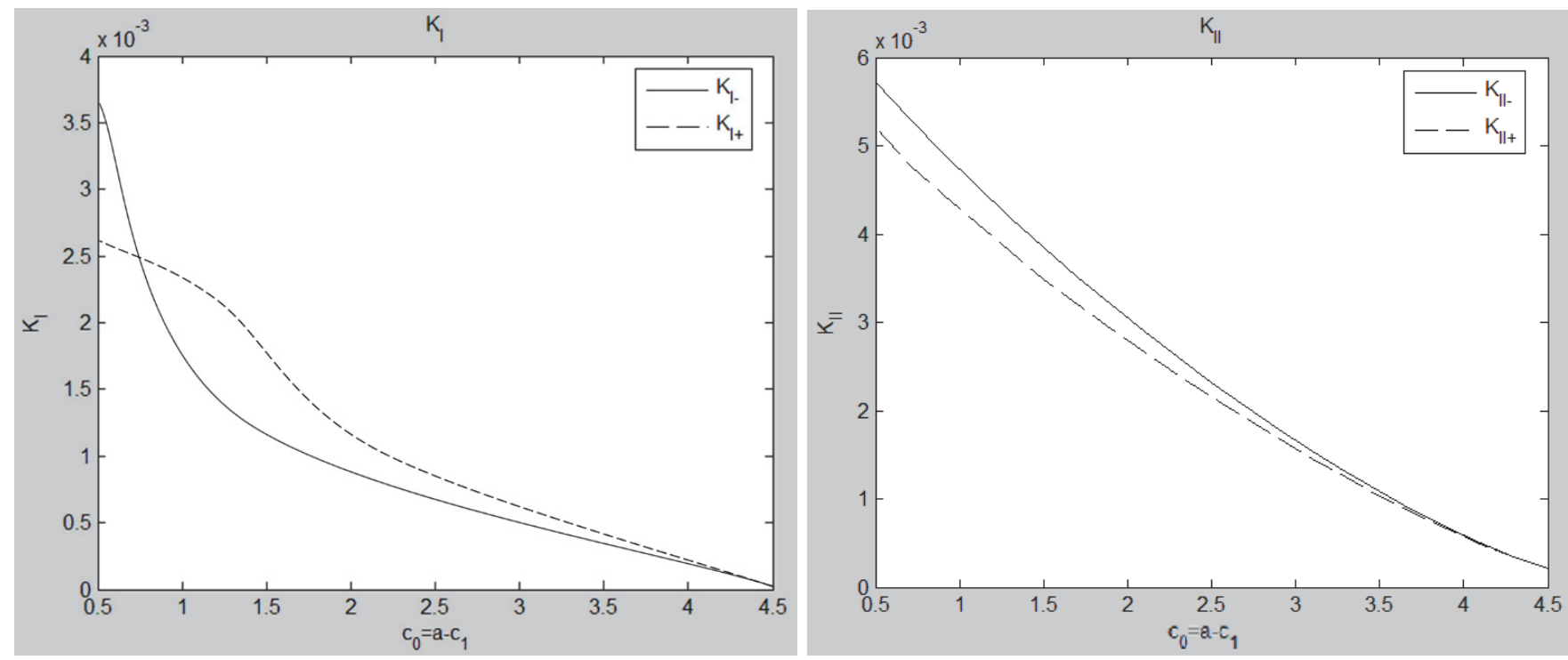

Figure 4: First case: The changing SIF $K_{I}, K_{I I}$ when the crack's size is increasing.

\section{CONCLUSIONS}

1 . The proposed method enhances the solution of the problem in two different cases of external load when the transverse crack is located inside the semi-strip.

2. The minimal distances between the crack's tips and the lateral sides of the semi-strip are established. The consideration of the fixed singularities at the semi-strip's short edge allows bringing the crack to the lateral sides more than $6 \%$ closer in comparison with the case when the fixed singularities were not considered. To obtain stable results when the crack is closer to the lateral sides one has to take into account the fixed singularities at the crack's tips.

3. The approach described the solution of the problem that can be also applied in the case of a dynamic statement of the problem.

\section{APPENDIX A. SOLVING THE MIXED PROBLEM OF ELASTICITY FOR A QUARTER PLANE}

$\mathrm{C}$ onsider the problem for a quarter plane (Fig. 5) $x, y>0$ when one boundary side $x=0,0<y<\infty$ is fixed and the another $y=0,0<x<\infty$ is under the mechanical load $r(x)=\left\{\begin{array}{ll}p(x) & 0<x<a, \\ 0, & x>a\end{array}\right.$.

The initial problem was reduced to a one-dimensional problem with the help of the semi-infinite sin-, cos- Fourier transformation (9). The boundary problem in transformation domain was rewritten in the vector form

$$
\begin{aligned}
& L_{2} \vec{y}_{\beta}(x)=\vec{g}(x) \\
& \vec{y}_{\beta}(0)=0
\end{aligned}
$$




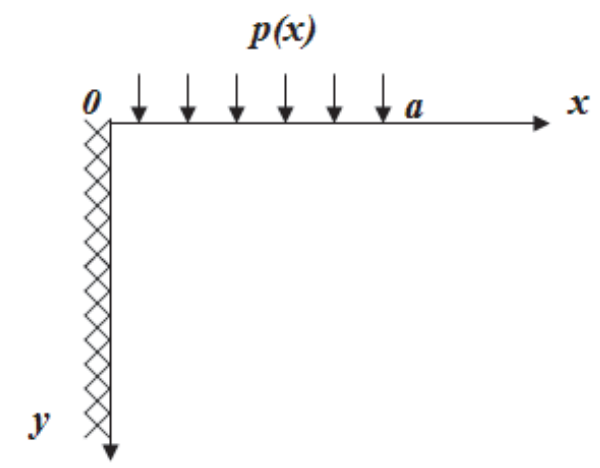

Figure 5: Geometry and coordinate system of the quarter plane.

here

$$
\begin{aligned}
& L_{2} \vec{y}_{\beta}(x)=I_{\beta}{ }^{\prime \prime}(x)+2 \beta Q \vec{y}_{\beta}{ }^{\prime}(x)-\beta^{2} P \vec{y}_{\beta}(x) \\
& I \text { is an identity matrix, } \vec{y}_{\beta}(x)=\left(\begin{array}{l}
u_{\beta}(x) \\
v_{\beta}(x)
\end{array}\right) \\
& P=\left(\begin{array}{cc}
\frac{\kappa-1}{\kappa+1} & 0 \\
0 & \frac{\kappa+1}{\kappa-1}
\end{array}\right) \\
& Q=\left(\begin{array}{cc}
0 & \frac{1}{\kappa+1} \\
-\frac{1}{\kappa-1} & 0
\end{array}\right) \\
& \vec{g}(x)=\left(\begin{array}{c}
\frac{3-\kappa}{\kappa+1} \chi^{\prime}(x) \\
-\beta \frac{\kappa+1}{\kappa-1} \chi(x)
\end{array}\right)
\end{aligned}
$$

The new unknown function is input $\chi(x)=\left.v(x, y)\right|_{y=0}$.

The solution of (17) was constructed in the following form

$$
\vec{y}_{\beta}(x)=\tilde{Y}(x)\left(\begin{array}{c}
\tilde{c}_{1} \\
\tilde{c}_{2}
\end{array}\right)+\int_{0}^{\infty} \tilde{G}(x, \xi) \vec{g}(\xi) d \xi
$$

where

$$
\tilde{Y}(x)=\frac{e^{-\beta x}}{2}\left(\begin{array}{cc}
-\frac{\kappa+\beta x}{\beta(\kappa-1)} & \frac{x}{\kappa+1} \\
-\frac{x}{\kappa-1} & -\frac{\kappa-\beta x}{\beta(\kappa+1)}
\end{array}\right)
$$


was constructed with the help of the matrix differential calculation, $\tilde{c}_{i}, i=1,2$ are known constants, $\tilde{G}(x, \xi)$ is the Green's matrix function which was constructed by the use of the matrix semi-infinite Fourier transformation. The components of the Green's matrix function have the following form

$$
\begin{aligned}
& \tilde{G}_{11}(x, \xi)=\frac{1}{2 \beta}\left(e^{-\beta(x+\xi)}-e^{-\beta|x-\xi|}\right)+\frac{1}{2(\kappa-1)}\left(\frac{e^{-\beta(x+\xi)}-e^{-\beta|x-\xi|}}{\beta}+(x+\xi) e^{-\beta(x+\xi)}-|x-\xi| e^{-\beta|x-\xi|}\right) \\
& \tilde{G}_{12}(x, \xi)=\frac{1}{2(\kappa+1)}\left((x+\xi) e^{-\beta(x+\xi)}+(x-\xi) e^{-\beta|x-\xi|}\right) \\
& \tilde{G}_{21}(x, \xi)=\frac{1}{2(\kappa-1)}\left((x+\xi) e^{-\beta(x+\xi)}-(x-\xi) e^{-\beta|x-\xi|}\right) \\
& \tilde{G}_{22}(x, \xi)=-\frac{1}{2 \beta}\left(e^{-\beta(x+\xi)}+e^{-\beta|x-\xi|}\right)+\frac{1}{2(\kappa+1)}\left(\frac{e^{-\beta(x+\xi)}+e^{-\beta|x-\xi|}}{\beta}+(x+\xi) e^{-\beta(x+\xi)}+|x-\xi| e^{-\beta|x-\xi|}\right)
\end{aligned}
$$

After inverting the expression (18), and the summation of the weak-convergent integrals, the formulae for the displacements in the quarter plane have the following form

$$
\begin{aligned}
& u(x, y)=\frac{2}{\pi} \int_{0}^{\infty} \chi^{\prime}(\xi)\left\{\frac{\kappa-1}{4(\kappa+1)}\left[\ln \left((\xi+x)^{2}+y^{2}\right)-\ln \left((\xi-x)^{2}+y^{2}\right)\right]+\frac{1}{\kappa+1}\left[\frac{(\xi-x)^{2}}{(\xi-x)^{2}+y^{2}}-\frac{(\xi+x)^{2}}{(\xi+x)^{2}+y^{2}}\right]-\right. \\
& \left.-\frac{x}{\kappa(\kappa+1)}\left[\frac{2 \xi\left((\xi+x)^{2}-y^{2}\right)}{\left((\xi+x)^{2}+y^{2}\right)^{2}}-\frac{(\xi+x)(\kappa+1)}{(\xi+x)^{2}+y^{2}}\right]\right\} d \xi \\
& v(x, y)=-\frac{2}{\pi} \int_{0}^{\infty} \chi^{\prime}(\xi)\left\{\frac{1}{2 \kappa+2}\left[\frac{y(\xi-x)}{(\xi-x)^{2}+y^{2}}+\frac{y(\xi+x)}{(\xi+x)^{2}+y^{2}}\right]-\frac{1}{2}\left[\operatorname{arctg}\left(\frac{y}{\xi+x}\right)-\operatorname{sign}(x-\xi) \operatorname{arctg}\left(\frac{y}{|x-\xi|}\right)\right]+\right. \\
& \left.+2 \operatorname{arctg}\left(\frac{y}{\xi+x}\right)+\frac{2}{\kappa(\kappa+1)}\left[\frac{4 \xi x y(\xi+x)}{\left((\xi+x)^{2}+y^{2}\right)^{2}}-\frac{x y(\kappa+1)}{(\xi+x)^{2}+y^{2}}-\frac{2 \kappa \xi y}{(\xi+x)^{2}+y^{2}}\right]\right\} d \xi+\chi(x) \operatorname{sign}(y)
\end{aligned}
$$

These expressions will describe the displacements in the quarter plane if the function $\chi^{\prime}(\xi)$ is found. To get it, the formulae for the displacements were put in the boundary condition $\sigma_{y}(x, 0)=p(x), 0<x<a$. After changing the variables, the singular integral equation was derived

$$
\int_{0}^{1} \tilde{\chi}(\xi)\left[\frac{1}{\xi-x}+\frac{h_{1}}{\xi+x}+\frac{h_{2} x}{(\xi+x)^{2}}+\frac{h_{3} \xi x}{(\xi+x)^{3}}\right] d \xi=q(x)
$$

here $\tilde{\chi}(\xi)=\chi^{\prime}\left(\frac{a(\xi+1)}{2}\right), h_{1}=-\frac{\kappa^{2}-3}{2 \kappa}, h_{2}=-\frac{2}{\kappa}, h_{3}=\frac{4}{\kappa}, q(x)$ is the known function. 
The transcendental equation for (19) is equal to the transcendental equation for the first singular integral equation in (13), and has the following form

$$
\cos (\pi \lambda)+\frac{2 \lambda^{2}}{3-4 \mu}-\frac{4 \lambda}{3-4 \mu}-\frac{8 \mu^{2}-12 \mu+3}{3-4 \mu}=0
$$

The Eq. (20) is equal to the transcendental equation in [32].

\section{ACKNOWLEDGMENTS}

he authors are grateful to Simon Dyke for the editing of the manuscript's text.

\section{REFERENCES}

[1] Vorovich, I. I. and Kopasenko, V. V., (1966). Some problems of elasticity theory for the semi-strip. (in Russian), Prikladnaya matematica i mekchanica, 30(1), pp. 128-136.

[2] Babeshko, V. A., Babeshko, O. M. and Evdokimova, O. V., (2010). On the method of block element, Mechanics of Solids, 45, pp. 437-444. DOI: 10.3103/S0025654410030143.

[3] Duduchava, R. V., (1979). Integral equations with fixed singularities, BG Teubner.

[4] Antipov, Y. A., (2015). Singular integral equations with two fixed singularities and applications to fractured composites, The Quarterly Journal of Mechanics and Applied Mathematics, 68(4), pp. 461-501.

[5] Onischuk, O. V., Popov, G. Ya. and Farshayt, P. G., (1988). The problem about bend of rectangular plate with linear pile, which goes on the fixed side by one end, Mechanics of solids, 6, pp. 160-167.

[6] Soldatov, A. P., (1973). A problem in function theory, Diff. Equations, 9(2), pp. 248-253.

[7] Bueekner, H. F., (1960). Some stress singularities and their computation by means of integral equations, Boundary problems in differential equation, Univ. Wisconsin Press. Madison, pp. 215-230.

[8] Bueekner, H. F., (1966). On a class of singular integral equations, J. Mathem. Anal. and Appl., 14, pp. 392-426.

[9] Bierman, G. I., (1971). A particular class of singular integral equations, J. Appl. Mathem., 20(1), pp. 99-109.

[10] Gohberg, I. and Krein, M.G., (1960). Introduction of the Theory of Linear nonselfadjoint operators, American mathematical society. Transl, 14, pp. 217-287.

[11] Noble, B., (1958). Methods Based on the Wiener-Hopf Technique For the Solution of Partial Differential Equations, Belfast, Northern Ireland, Pergamon Press.

[12] Tricomi, F., (1932). Atti Accad. Naz. Lincei, Ser. 5(14), pp. 134-247.

[13] Michlin, S. G. and Prössdorf, S., (1986). Singular Integral Operators, Akademie Verlag, Berlin.

[14] Wu X.-F., Lilla, E. and Zou, W.-S., (2002). A semi-infinite internal crack between two bonded dissimilar elastic strips, Archive of Applied Mechanics, 72, pp. 630-636.

[15] Duduchava, R., Krupnik, N. and Shargorodsky, E., (1999). An algebra of integral operators with fixed singularities in kernels, Integral Equations and Operator Theory, 33(4), pp. 406-425.

[16] Junghanns, P. and Rathsfeld, A., (2002). On polynomial collocation for Cauchy singular integral equations with fixed singularities, Integral Equations and Operator Theory, 43(2), pp. 155-176.

[17] Savruk, M. P., Osiv, P. N. and Prokopchuk, I. V., (1989). Numerical analysis in plane problems of the crack's theory (in Russian), Naukova dumka, Kyiv.

[18] Popov, V. G., (2012). A dynamic contact problem which reduces to a singular integral equation with two fixed singularities, Journal of Applied Mathematics and Mechanics, 76(3), pp. 348-357.

[19] Shabozov, M. Sh., (1995). An approach to the investigation of optimal quadrature formulas for singular integrals with fixed singularity, Ukrainian Mathematical Journal, 47(9), pp. 1479-1485.

[20] Sahakyan, A. V., (2011). Method of discrete singularities for solution of singular integral and integro-differential equations, Proceedings of A. Razmadze Mathematical Institute, 156. 
[21] Gabbasov, N. S., (2009). Methods for solving an integral equation of the third kind with fixed singularities in the kernel, Differential Equations, 45(9), pp. 1341-1348.

[22] Capobianco, M. R., Criscuolo, G. and Junghanns, P., (2008). On the numerical solution of a hypersingular integral equation with fixed singularities, Operator Theory: Advances and Applications, 187, pp. 95-116.

[23] Guz, A.N., Guz, I.A., Men'shikov, A.V. and Men'shikov, V.A., (2011). Stress-intensity factors for materials with interface cracks under harmonic loading, Int Appl Mech, 46, pp. 1093. DOI: 10.1007/s10778-011-0401-1.

[24] Di Cocco, V. and Iacoviello, F., (2017). Ductile cast irons: Microstructure influence on the damaging micromechanisms in overloaded fatigue cracks, Engineering Failure Analysis, 82, pp. 340-349.

[25] Toribio, J., Gonzàles, B. and Matos, J.C., (2017). Crack tip field in circumferentially-cracked round bar (CCRB) in tension affected by loss of axial symmetry, Frattura ed Integrità Strutturale, 41, pp. 139-142, DOI: 10.3221/IGFESIS.41.19.

[26] Peron, M., Razavi, S.M.J., Berto, F. and Torgersen, J., (2017) Notch stress intensity factors under mixed mode loadings: an overview of recent advanced methods for rapid calculation, Frattura ed Integrità Strutturale, 42, pp. 196-204, DOI: 10.3221/IGF-ESIS.42.21.

[27] Tokovyy, Yu. and Chien-Ching Ma, (2009). An explicit-form solution to the plane elasticity and thermoelasticity problems for anisotropic and inhomogeneous solids, International Journal of Solids and Structured, 46, pp. 3850-3859.

[28] Vaysfel'd, N., Kryvyi, O. and Zhuravlova, Z., (2016). On the stress investigation at the edge of the fixed elastic semistrip, Frattura ed Integrità Strutturale, 38, pp. 1-11. DOI: 10.3221/IGF-ESIS.38.01.

[29] Vaysfel'd, N. D. and Zhuravlova, Z. Yu., (2015). On one new approach to the solving of an elasticity mixed problem for the semi-strip, Acta Mechanica, 226(12), pp. 4159-4172. DOI: 10.1007/s00707-015-1452-x.

[30] Popov, G.Ya., (1982). The elastic stress' concentration around dies, cuts, thin inclusions and reinforcements(in Russian), Nauka, Moskow.

[31] Vaysfel'd, N.D. and Zhuravlova Z.Yu., (2018). Two-dimensional mixed problem of thermoelasticity for a semistrip, Journal of Mathematical Sciences, 228(2) 105-121. DOI 10.1007/s10958-017-3609-8.

[32] Uflyand, Ya. S., (1967). Integral transformations in the problems of the elasticity theory (in Russian), Nauka, L..

[33] Zhuravlova, Z., (2017). Stress analysis near the tips of a transverse crack in an elastic semi-strip, Appl. Math. Mech. Engl. Ed. DOI: 10.1007/s10483-017-2217-6. 